\title{
Finite horizon regulator problem: the non-standard case
}

\author{
Francesca Bucci* \\ Università di Firenze, Dipartimento di Matematica Applicata \\ Via S. Marta 3, 50132 Firenze, Italy. Phone: +39-11-4796248. \\ $E$-mail fbucci@ing.unifi.it \\ and \\ Luciano Pandolfi \\ Politecnico di Torino, Dipartimento di Matematica \\ Corso Duca degli Abruzzi, 24, 10129 Torino, Italy. \\ Phone: +39-11-5647516. \\ $E$-mail lucipan@polito.it
}

\begin{abstract}
We present several regularity properties of the value function of a quadratic control problem. The system is distributed, with distributed control action while the quadratic functional is not coercive; even it may not be positive.
\end{abstract}

\section{Keywords}

Quadratic regulator problem, Dissipative system, Singular control

\section{INTRODUCTION}

We present in this talk several new results about the finite horizon non-standard quadratic regulator problem for a distributed system with bounded input operator.

In this introduction we recall the main known results for finite dimensional systems, a case in which the theory is complete; and we mention the few known extensions to the distributed case.

The system that we intend to study is described by the equation

$\dot{x}=A x+B u, \quad x(\tau)=x_{0}$.

\footnotetext{
* Partially supported by the Italian Ministero della Ricerca Scientifica e Technologica within the program of GNAFA-CNR.

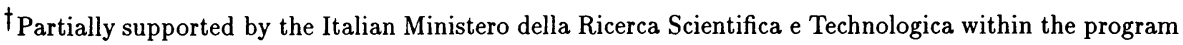
of GNAFA-CNR and by HCM network CEC n. ERB-CHRX-CT93-0402.
} 
Here $\tau$ is a point which belongs to the interval $[0, T)$ and we solve equation (1) on the interval $[\tau, T]$.

The (mild) solution to Eq. (1) is given by

$$
x(t)=x\left(t ; \tau, x_{0}, u\right)=e^{A(t-\tau)} x_{0}+\int_{\tau}^{t} e^{A(t-s)} B u(s)=e^{A(t-\tau)} x_{0}+\left(L_{\tau} u\right)(t)
$$

for each square integrable control $u(\cdot)$. Here $e^{A s}$ is a $C_{0}$-semigroup on a Hilbert space $X$ whose generator is $A ; B \in \mathcal{L}(U, X)$. The space $U$ is a second Hilbert space.

We associate the following quadratic functional to the system described by (1):

$J_{\tau}\left(x_{0}, u\right)=\int_{\tau}^{T} F(x(t), u(t)) d t+\left\langle x(T), P_{0} x(T)\right\rangle$,

where $x=x(t)=x\left(t ; \tau, x_{0}, u\right)$ and $F(x, u)=\langle x, Q x\rangle+\langle u, R u\rangle$. The operators $Q, R$ and $P_{0}$ are linear bounded operators in the proper spaces, $R$ and $P_{0}$ are selfadjoint. We do not make any assumption on their definiteness, in contrast with the so called standard case, when

$Q \geq 0, \quad R \geq \alpha>0, \quad P_{0} \geq 0$.

The standard regulator problem is completely understood and the solution is reduced to the study of an operator Riccati equation. Its solution provides the synthesis of the unique optimal control. In contrast with this, there will be no optimal control in the non standard case and, correspondingly, no Riccati equation.

We note that the same results we are going to describe below hold even if $F$ presents a mixed term in $x$ and $u$.

Our goal is to characterize the following property

$$
\text { Property } 1 V_{\tau}\left(x_{0}\right):=\inf _{u \in L^{2}(\tau, T ; U)} J_{\tau}\left(x_{0}, u\right)>-\infty \quad \forall x_{0} \in X \quad \forall \tau \in[0, T] .
$$

If this condition holds, we want to study the regularity properties of the function $\tau \rightarrow$ $V_{\tau}\left(x_{0}\right)$ for any specified $x_{0} \in X$.

The non-standard - or singular - LQR problem was completely solved in finite dimension (see in particular Clements and Anderson (1978), Molinari (1975)). These authors were stimulated mostly by the analysis of the second variations of non-linear optimization problems. These problems are obtained computing the system along a candidate optimal solution, so that in general the matrices $A, B, Q, R$ are time-dependent. Here we confine ourselves to the time-invariant case.

The main results that have been obtained are as follows:

When Property 1 holds, then for each $\tau$ the functional $x_{0} \rightarrow V_{\tau}\left(x_{0}\right)$ is a (continuous) quadratic functional, namely

$V_{\tau}\left(x_{0}\right)=\left\langle x_{0}, W(\tau) x_{0}\right\rangle$,

where $W(\tau)$ is a matrix of the same dimension as $x$. In general the dependence on $\tau$ is not "regular" as the following example from Clements and Anderson (1978) shows. 
Consider the dynamic $\dot{x}=u$, with cost given by

$J_{\tau}\left(x_{0}, u\right)=-\int_{\tau}^{1} k(t) x(t) u(t) d t+x^{2}(1) / 2$,

where $k(t)=0$ on $[0,1 / 2), k(t)=1$ on $[1 / 2,1]$. The value function $V_{\tau}\left(x_{0}\right)$ is equal to 0 for $\tau<1 / 2$ and to $x_{0}^{2} / 2$ for $t \geq 1 / 2$.

In this example the discontinuity is produced by the coefficient $k(\cdot)$. However we shall see that (for distributed parameter systems) we can have discontinuities even for timeinvariant systems.

A second property that it is proved for finite dimensional systems is the Dissipation Inequality, a certain inequality satisfied by $W(\cdot)$, which also holds for distributed parameter systems (see below). Actually, systems which enjoy this dissipation inequality have importance in physical applications since they correspond to systems which "dissipate energy" (compare Brune (1931), Willems (1972)). In fact the study of such dissipative systems-on $[0,+\infty)$-is a second problem which stimulated the analysis of the non-standard regulator problem. A third problem is the stability of Lur'e type feedback control systems. A very nice overview of the finite dimensional theory can be found in Bittanti, Laub, Willems (1991).

We stress that the previous results do not require that the cost $J_{\tau}\left(x_{0}, u\right)$ reaches a minimum value. We shall see that when a minimum is reached, more precise regularity properties on the function $\tau \rightarrow V_{\tau}\left(x_{0}\right)$ can be proved, see below.

Most of the proofs of the cited results, which all concern systems in $\mathrm{R}^{n}$, are not readily adaptable to the case of distributed systems, since they often rely on special features of finite dimensional systems, such as group property of the evolution operator, exact controllability, etc.

For completeness we add that the distributed parameters time varying case was studied by Jacob (1995), under the restriction that the operator $R$ is the identity operator. This paper shows, in particular, that the existence of the optimal control and the existence of solutions to the Riccati equation are related but not equivalent properties. In contrast with this paper by Jacob (1995) we are interested in the time-invariant case, with special emphasis in non-coercive quadratic functional.

The existing results for finite dimensional systems - infinite horizon case - were extended to the distributed control case in Louis and Wexler (1991).

\section{MAIN RESULTS}

In this section we describe our results. Some examples and proofs are sketched in the next section.

Theorem 1 Let $\tau \in[0, T]$. If

$V_{\tau}\left(x_{0}\right)>-\infty \quad \forall x_{0} \in X$, 
there exists a selfadjoint operator $W(\cdot) \in \mathcal{L}(X)$ such that $W(T)=P_{0}$ and

$V_{r}\left(x_{0}\right)=\left\langle x_{0}, W(\tau) x_{0}\right\rangle$

We consider now the dissipation inequality.

Theorem 2 Property 1 holds if and only if for each $\tau \in[0, T]$ there exist a linear bounded operator $W(\tau) \in \mathcal{L}(X)$ which satisfies, for any $t \geq \tau$, the following dissipation inequality

$$
\langle x(t), W(t) x(t)\rangle-\langle x(\tau), W(\tau) x(\tau)\rangle+\int_{\tau}^{t} F(x(s), u(s)) \geq 0, \quad W(T) \leq P_{0} .
$$

In the above inequality $u(\cdot)$ is any admissible control, $x(t)=x\left(t ; \tau, x_{0}, u\right)$. Moreover, the inequality becomes an equality if and only if the control $u$ in (7) is optimal.

If the previous inequality holds true, then there exists a solution $P(t)$ of $(7)$ such that $V_{\tau}\left(x_{0}\right)=\left\langle x_{0}, P(\tau) x_{0}\right\rangle$.

Corollary 3 If for some number $\tau$ we have $V_{\tau}\left(x_{0}\right)>-\infty$ then we have also $V_{t}(x(t))>$ $-\infty$ for each $t \in[\tau, T]$. Here, $x(t)$ denotes the value at time $t$ of the function in (2), for any fixed control $u(\cdot)$ on $[\tau, t]$.

Examples in the next section will show that the function $\tau \rightarrow V_{\tau}\left(x_{0}\right)$ may not be continuous. ¿From this point of view, the following result have an interest.

Theorem 4 Let us assume that the point $x_{0}$ has the following property: $\tau \rightarrow V_{\tau}\left(x_{0}\right)$ is finite on $[0, T]$. Then

- the function $\tau \rightarrow V_{\tau}\left(x_{0}\right)$ is upper semicontinuous on $[0, T]$;

- the function $\tau \rightarrow V_{\tau}\left(x_{0}\right)$ is lower semicontinuous at $\tau_{0}$ provided that for each sequence $\tau_{n}$ which tends monotonically to $\tau_{0}$ there exists a sequence $\left\{u_{n}\right\} \in L^{2}\left(\tau_{n}, T ; U\right)$ such that

i) $\quad V_{\tau_{n}}\left(x_{0}\right) \leq J_{\tau_{n}}\left(x_{0} ; u_{n}\right) \leq V_{\tau_{n}}\left(x_{0}\right)+\frac{1}{n}$
ii) $\exists \gamma_{0}>0: \quad\left\|u_{n}(\cdot)\right\|_{L^{2}\left(\tau_{n}, T ; U\right)} \leq \gamma_{0}$.

Of course the previous choice for the properties of $u_{n}(\cdot)$ is suggested by the construction of minimizing sequences. And it may be easier to test the required condition in the case that an optimal control exists for each $\tau$ near $\tau_{0}$.

We shall see that lower semicontinuity needs not hold in all cases.

We now recall the following general result:

Lemma 5 Consider the functional

$J_{y}(\zeta)=2 \operatorname{Re}\langle\mathcal{N} y, \zeta\rangle+\langle\zeta, \mathcal{R} \zeta\rangle$

over a Hilbert space $Z$ (it will be $Z=L^{2}(\tau, T ; U$ ) in the following). If there exists $y$ such that $\inf _{\zeta} J_{y}(\zeta)>-\infty$, then $\mathcal{R} \geq 0$; moreover the functional $J_{y}(\zeta)$ admits at least one 
minimum on $Z$ for any fixed $y$ if and only if the following conditions are satisfied:

i) $\quad \mathcal{R} \geq 0 ; \quad$ ii) im $\mathcal{N} \subseteq$ im $\mathcal{R}$.

If the previous conditions hold, then any $\zeta$ such that $\mathcal{N} y+\mathcal{R} \zeta=0$ is a minimum.

In our application the operators $\mathcal{N}$ and $\mathcal{R}$ will be the operators given by

$$
\begin{aligned}
& \left(\mathcal{N}_{\tau} x\right)(t)=\left(L_{\tau}^{*} Q e^{A(\cdot-\tau)} x\right)(t)+S^{*} e^{A(t-\tau)} x+\left(L_{\tau, T}^{*} P_{0} e^{A(T-\tau)} x\right)(t) \\
& \left(\mathcal{R}_{\tau} u\right)(t)=\left(L_{\tau}^{*} Q\left(L_{\tau} u\right)\right)(\cdot)+S^{*}\left(L_{\tau} u\right)(t)+\left(L_{\tau}^{*} S u\right)(t)+R u(t)+\left(L_{\tau, T}^{*} P_{0} L_{\tau, T} u\right)(t)
\end{aligned}
$$

respectively.

The following result is crucial:

Theorem 6 If there exists $\tau_{0} \in[0, T]$ such that

$\mathcal{R}_{\tau_{0}} \geq \gamma$

then $R \geq \gamma$.

Of course the most important cases are $\gamma=0$ and $\gamma>0$ (coercive operator).

We note that this result is well known in the case that $T=+\infty$. But in this last case it is proved thanks to the frequency domain inequality. The frequency domain inequality is essentially related to the Parseval equality for Fourier transform so that it has no simple correspondence in the finite-horizon case.

If $\mathcal{R}_{\tau_{0}}$ is coercive, then an optimal control exists, and the control is unique (even if the quadratic form $F$ of the cost is not positive). Thanks to this property, the value function $V_{\tau_{0}}\left(x_{0}\right)$ displays better regularity properties. More precisely, we have the following

Theorem 7 Assume that there exists $\tau_{0}$ and $\gamma>0$ such that $\mathcal{R}_{\tau_{0}} \geq \gamma$. Then, for any $\tau \in\left[\tau_{0}, T\right]$,

- $\tau \rightarrow V_{\tau}(x)$ is continuous for any $x \in X$;

- $\tau \rightarrow V_{\tau}(x)$ is differentiable for any $x \in D(A)$.

In this case it is possible to deduce a differential form of the dissipation inequality.

Theorem 8 Assume that $\mathcal{R}_{\tau_{0}} \geq \gamma>0$ holds true. Then there exists a selfadjoint operator $W(\cdot) \in L(X)$ such that

i) $W(T)=P_{0}$;

ii) $W(\cdot)$ satisfies

$$
\begin{aligned}
& \frac{d}{d \tau}\langle a, W(\tau) a\rangle+2 R e\langle A a+B u, W(\tau) a\rangle+F(a, u) \geq 0, \\
& \text { for any }(a, u) \in D(A) \times U, \text { for any } \tau \in\left(\tau_{0}, T\right) .
\end{aligned}
$$




\section{EXAMPLES AND SKETCH OF THE PROOFS}

\subsection{Examples}

We present a first example which shows that the value function $V_{\tau}\left(x_{0}\right)$ may not be continuous at the final time $T$.

Example 1 Minimize the functional

$$
J_{\tau}\left(x_{0}, u\right)=\left\langle x(T), P_{0} x(T)\right\rangle, \quad P_{0}>0,
$$

with $x(\cdot)$ subject to any system (finite or infinite dimensional) which is null-controllable from any state in an arbitrary time. It is readily verified that $V_{T}\left(x_{0}\right)=\left\langle x_{0}, P_{0} x_{0}\right\rangle$, while $V_{\tau}\left(x_{0}\right)=0$ for any $\tau<T$, hence $V_{\tau}\left(x_{0}\right)$ is discontinuous in $T$ except when $x_{0}=0$.

Example 2 Consider the dynamic

$$
\begin{cases}\dot{x}(t) & =\xi(t,-1)+u(t) \\ \frac{d}{d t} \xi(t) & =A \xi-w(t) \\ \dot{u}(t) & =w(t)\end{cases}
$$

on the state space $\mathrm{R} \times L^{2}(-1,0) \times \mathrm{R}$. The operator $A$ is defined by

$$
D(A)=\left\{\xi \in H^{1}([-1,0]), \xi(0)=0\right\} \quad(A \xi)(\theta)=\frac{\mathrm{d}}{\mathrm{d} \theta} \xi(\theta)
$$

It is easily seen that any solution $t \rightarrow(x(t), \xi(t, \theta), u(t))$ is equal to $t \rightarrow(x(t), u(t+\theta)-$ $u(t), u(t))$, where $x(t)$ satisfies the input delay equation

$\dot{x}=u(t-1)$,

provided that $u(\cdot)$ is differentiable. This shows that system (14) defines a $C_{0}$-semigroup on the space $\mathrm{R} \times L^{2}(-1,0) \times \mathrm{R}$.

We now study system (14) on the interval $[\tau, 2]$ and we associate to it the following cost:

$J\left(x_{0}, \xi_{0}, u_{0} ; w\right)=|x(2)|^{2}$.

Consider the problem with $x_{0}=x(0) \neq 0, \xi_{0}(\theta) \equiv 0$, and $u_{0}=0$. Controls $u(\cdot)$ are taken to be zero out of $[\tau, T]$.

It is seen from (15) that if $1<\tau<2$ then $x(\cdot)$ is not affected by $u(\cdot)$ (i.e. by $w(\cdot))$. Hence, $x(t) \equiv x_{0}$ and $V_{\tau}\left(x_{0}\right)=\inf _{w} J\left(x_{0}, 0,0 ; w\right) \equiv\left|x_{0}\right|^{2}$ on $[1,2]$.

If $\tau \in(0,1)$ then the function $x(\cdot)$ is affected by the restriction of $u(\cdot)$ to the interval $[\tau, 1]$ and this function can be arbitrarily selected, within the set of $H^{1}$ functions which are zero at $\tau$, by a suitable choice of $w(\cdot)$. Hence we can use the control on $[\tau, 1]$ in order to have $x(1)=0$ i.e. $x(t) \equiv 0$ on [1,2]: $V_{\tau}\left(x_{0}\right)=0$ for $\tau<1$. In conclusion, the function $\tau \rightarrow V_{\tau}\left(x_{0}\right)$ is discontinuous at $\tau_{0}=1$. 
Remark The previous example shows that in the statement of Theorem \& concerning lower semicontinuity of $V_{\tau}\left(x_{0}\right)$, assumption ii) cannot be dispensed with. Notice that in fact ii) holds for $\tau \rightarrow 1^{+}$, but not for $\tau \rightarrow 1^{-}$.

\subsection{Sketch of some proofs}

Proof. of Theorem 4: Fix $x_{0} \in X$, and let $\tau_{0} \in(0, T)$. In order to show upper semicontinuity, we shall show that for any real number $\alpha>V_{\tau_{0}}\left(x_{0}\right)$ we have $\alpha>V_{\tau}\left(x_{0}\right)$, if $\left|\tau-\tau_{0}\right|$ is taken small enough. Consider the case when $\tau>\tau_{0}$. Let $u$ be an admissible control such that $J_{\tau_{0}}\left(x_{0}, u\right)<\alpha$, and define $x_{\tau}(t)=e^{A(t-\tau)} x_{0}+\int_{\tau}^{t} e^{A(t-s)} B u(s)$. It is readily verified that

1. $\lim _{\tau \rightarrow \tau_{0}} x_{\tau}(T)=x_{\tau_{0}}(T)$,

2. $\lim _{\tau \rightarrow \tau_{0}} \int_{\tau}^{T} F\left(x_{\tau}(s), u(s)\right)-\int_{\tau_{0}}^{T} F\left(x_{\tau_{0}}(s), u(s)\right)=0$,

so that if $\left|\tau-\tau_{0}\right|$ is small enough, $V_{\tau}\left(x_{0}\right) \leq J_{\tau}\left(x_{0}, u\right)<\alpha$. The case $\tau<\tau_{0}$ is similar.

As to lower semicontinuity, we consider, for $\tau_{n} \downarrow \tau_{0}$, the sequence

$\hat{u}_{n}(s):= \begin{cases}0 & \tau_{0}<s<\tau_{n} \\ u_{n}(s) & s \geq \tau_{n} .\end{cases}$

By assumption $(8 i i)$ it follows that $\hat{u}_{n}(\cdot) \rightarrow v(\cdot) \in L^{2}\left(\tau_{0}, T ; U\right)$, and moreover

$\int_{\tau_{0}}^{T} F\left(x\left(t ; \tau_{0}, x_{0}, \hat{u}_{n}\right), \hat{u}_{n}(t)\right) d t-\int_{\tau_{n}}^{T} F\left(x\left(t ; \tau_{n}, x_{0}, u_{n}\right), u_{n}(t)\right) d t \rightarrow 0$.

Therefore, by convexity of the cost, and due to the dominated convergence theorem,

$V_{\tau_{0}}\left(x_{0}\right) \leq J_{\tau_{0}}\left(x_{0}, v\right) \leq \liminf _{n \rightarrow \infty} J_{\tau_{0}}\left(x_{0}, \hat{u}_{\tau_{n}}\right)=\liminf _{n \rightarrow \infty} V_{\tau_{n}}\left(x_{0}\right)$.

If $\tau \uparrow \tau_{0}$, define instead $\hat{u}_{n}(s)=u_{n}(s)_{\mid\left[\tau_{0}, T\right]}$. We omit details.

Proof. of Theorem 6 We note preliminarly that if $\tau>\tau_{0}$, we can write

$\left\langle\mathcal{R}_{\tau} u, u\right\rangle_{L^{2}(\tau, T ; U)}=\left\langle\mathcal{R}_{\tau_{0}} v, v\right\rangle_{L^{2}\left(\tau_{0}, T ; U\right)}$,

where $v(\cdot)$ is

$v(t)= \begin{cases}0 & \tau \leq t<\tau \\ u(t) & t \geq \tau\end{cases}$

Hence, from (12) it follows that

$\mathcal{R}_{\tau} \geq \gamma \quad \forall \tau \in\left[\tau_{0}, T[\right.$. 
We first consider the case $\alpha=0$, hence by assumption $\mathcal{R}_{\tau_{0}} \geq 0$. By contradiction, suppose that there exists a control $u_{0} \in U$ and a constant $\alpha>0$ such that $\left\langle R u_{0}, u_{0}\right\rangle=-\alpha$. Given a small $\epsilon>0$, choose a control $u$ as follows:

$u(t)= \begin{cases}0 & \tau_{0} \leq t<T-\epsilon \\ u_{0} & T-\epsilon \leq t \leq T\end{cases}$

and compute

$$
\begin{aligned}
\left\langle\mathcal{R}_{\tau} u, u\right\rangle & =\int_{T-\epsilon}^{T}\left\langle Q \int_{T-\epsilon}^{t} e^{A(t-s)} B u_{0} d s, \int_{T-\epsilon}^{t} e^{A(t-s)} B u_{0} d s\right\rangle d t+\epsilon\left\langle R u_{0}, u_{0}\right\rangle+ \\
& +\int_{T-\epsilon}^{T}\left\langle P_{0} \int_{T-\epsilon}^{T} e^{A(T-s)} B u_{0} d s, \int_{T-\epsilon}^{T} e^{A(T-s)} B u_{0} d s\right\rangle d t=-\epsilon \alpha+o\left(\epsilon^{2}\right)+o\left(\epsilon^{3}{ }^{3} 17\right)
\end{aligned}
$$

Since $\epsilon$ can be taken arbitrarily small, (17) yields $\left\langle\mathcal{R}_{\tau_{0}} u, u\right\rangle<0$, and this contradicts the statement of Lemma 5 .

Assume instead (12) with $\gamma>0$. By choosing $u(t)=0$ for $t \in\left[\tau_{0}, T-\epsilon\left[, u(t)=u_{0} \in U\right.\right.$ arbitrary when $t \in[T-\epsilon, T]$, a direct computation yields

$\gamma \epsilon\left\|u_{0}\right\|^{2} \leq \epsilon\left\langle R u_{0}, u_{0}\right\rangle+o\left(\epsilon^{3}\right)\left\|u_{0}\right\|^{2}$

which implies $\left\langle R u_{0}, u_{0}\right\rangle \geq \gamma\left\|u_{0}\right\|^{2}$ for any $u_{0} \in U$.

\section{REFERENCES}

Bittanti, S., Laub, A.J., Willems, J.C. (1991) The Riccati equation, Springer-Verlag, Berlin.

Brune, O. (1931) Synthesis of a finite two terminal network whose driving point impedance is a prescribed function of impedancy, Journal of Mathematics \& Physics, 10 191-236.

Clements, D.J. and Anderson, B.D.O. (1978) Singular optimal control: the linearquadratic problem, Lecture Notes LNCIS no.5, Springer Verlag, Berlin.

Jacob, B. (1995) Linear quadratic optimal control of time-varying systems with indefinite costs on Hilbert spaces: the finite horizon problem, Journal of Mathematical Systems, Estimation 8 Control, 5 1-28.

Louis, J-Cl. and Wexler, D. (1991) The Hilbert space regulator problem and operator Riccati equation under stabilizability, Annales de la Société Scientifique de Bruxelles, 105 137-65.

Molinari, B.P. (1975) Nonnegativity of a quadratic functional, SIAM Journal Control 8 Optimization, 13 792-806.

Willems, J.C. (1972) Dissipative dynamical systems, Part I: general theory, Part II: Linear systems with quadratic supply rates, Arch. Rational Mechanics and Analysis, 45 321351 and $352-392$. 